Reprod. Nutr. Dévelop., 1981, 21 (2), 293-301.

\title{
Etude de la synthèse protéique in vivo dans le muscle de la truite arc-en-ciel (Salmo gairdneri R.). Influence de la température
}

\author{
par B. FAUCONNEAU, M. ARNAL *, P. LUQUET
}

Laboratoire de Nutrition des Poissons, INRA

Saint Pée sur Nivelle, 64310 Ascain, France

* Laboratoire d'Ełude du Métabolisme azoté, INRA

Theix, 63110 Beoumont, France.

Summary. In vivo protein synthesis in rainbow trout (Salmo gairdneri R.) muscle. Effect of temperafure acclimatization.

Protein synthesis was measured in the muscle of trout acelimatized to 10 and $18{ }^{\circ} \mathrm{C}$ and weighing about $100 \mathrm{~g}$. Fractional synthesis rate was calculated after a pulse injection of L-leucine $U^{14} \mathrm{C}$ by fitting the specific radioactivity of the protein to the computed values obtained with the specific radioactivity of the precursor, according to a precursor product relationship (Martin et al., 1977).

At 10 and $18^{\circ} \mathrm{C}$, fractional synthesis rate values were 0.9 and $1.2 \mathrm{p} .100 / \mathrm{d}$ (plasma as the precursor pool) and 1.9 and $1.8 \mathrm{p} .100 / \mathrm{d}$ (intracellular pool as the precursor), respectively.

Muscle protein synthesis rates in young trout are lower than in young mammals (rat, rabbit, lamb), and temperature acclimatization had no influence on muscle protein synthesis in trout.

\section{Introduction.}

A la suite de nombreuses études qualitatives de la synthèse protéique in vivo dans différents tissus des poissons, notamment dans le muscle (Das et Prosser, 1967 ; Jackim et Laroche, 1973 ; Somero ef Doyle, 1973 ; Smith et Haschemeyer, 1974 ; Narayansingh et Eales, 1975), des déterminations quantitatives du phénomène de renouvellement des protéines ont été entreprises à l'aide des méthodes utilisées chez les Mammifères (Waterlow, Garlick et Millward, 1978 ; Zak, Martin ef Blough, 1979). La méthode de décroissance appliquée chez la Carpe (Castilla, 1974 ; Bouche, 1975) et chez Gillichthys (Somero et Doyle, 1973) montre que la vitesse de dégradation des protéines dans le muscle de Poisson est légèrement plus lente que celle observée dans le foie et comparable à celle mesurée dans le muscle des Mammifères. La vitesse de synthèse des protéines musculaires mesurée à l'aide de la méthode d'infusion chez le muge et le baliste (Haschemeyer et Smith, 1979 ; Haschemeyer, Persell et Smith, 1979) est 20 à 40 fois plus lente que celles des protéines du foie et des branchies et 20 fois plus lente que celle des protéines du muscle de mammifères (muscle blanc). L'élévation 
de température augmente la quantité de protéines synthétisées dans chaque tissu, mais ne change pas de façon significative, l'écart entre les tissus (Haschemeyer, Persell et Smith, 1979). De telles disparités entre espèces, expliquées en partie par des différences de méthodes de mesure, pourraient aussi être la conséquence d'une liaison plus forte chez les poissons que chez les mammifères entre les phénomènes de synthèse des protéines et les processus de croissance.

Nous avons étudié la vitesse de synthèse des protéines du muscle de truife à l'aide de la méthode d'incorporation (Zak, Martin et Blough, 1979) après injection de leucine ${ }^{14} \mathrm{C}(U)$. L'étude a été réalisée sur des truites acclimatées à 10 et $18{ }^{\circ} \mathrm{C}$ afin de mettre en évidence dans les processus étudiés l'influence de la poecilothermie sur le métabolisme protéique du muscle et l'adaptation de ce métabolisme à la température.

\section{Matériel et méthodes.}

Deux lots de 30 truites arc-en-ciel de $80 \mathrm{~g}$ environ sont acclimatées pendant 3 à 4 semaines à 10 et $18^{\circ} \mathrm{C}\left( \pm 1^{\circ} \mathrm{C}\right)$ ef nourris à satiété au rythme de deux repas par jour avec un aliment (sous forme de granulés) contenant 52 p. 100 de protéines et 8 p. 100 de matières grasses (en p. 100 de la matière sèche) jusqu'au poids de $100 \mathrm{~g}$ environ. La croissance hebdomadaire (le poisson anesthésié par le monophényléther d'éthylène glycol en solution à 0,4 p. 100 dans l'eau, égoutté, est pesé dans un récipient contenant de l'eau et taré) ef la consommation journalière (contrôle du nombre de granulés consommés et refusés, rapporté à la quantité d'aliment distribué) sont mesurées individuellement.

La L-leucine ${ }^{14} \mathrm{C}(U)(328 \mathrm{mCi} / \mathrm{mmole})$ est injectée dans la veine caudale des truites anesthésiées à raison de $6,5 \mu \mathrm{Ci} / 100 \mathrm{~g}$ à $10^{\circ} \mathrm{C}$ et $7,2 \mu \mathrm{Ci} / 100 \mathrm{~g}$ à $18{ }^{\circ} \mathrm{C}$. Aux temps 30 , $60,120,240$ et $360 \mathrm{~min}$ et $20,40,90,180$ et $360 \mathrm{~min}$ après l'injection ef respectivement à 10 ef $18{ }^{\circ} \mathrm{C}$, les truites sonf abattues ( 5 truites/temps). Le sang est prélevé dans la veine caudale, hépariné, centrifugé à froid $\left(+4^{\circ} \mathrm{C}\right)$. Le muscle latéro dorsal est disséqué en partie ( $80 \mathrm{p} .100)$ puis plasma et muscle sont congelés dans l'azote liquide.

Les fractions acido-solubles (acide trichloracétique, TCA) et insolubles du plasma et du muscle sont obtenues par 4 extractions successives au TCA 10 p. 100 (Ferrara, 1976). Le TCA est éliminé sur résine anionique Dowex $2 \times 8$. L'insoluble est neutralisé (méthanol acétate) et dégraissé (méthanol chloroforme) afin d'obtenir un résidu protéique qui est hydrolysé par $\mathrm{HCl} 5,5 \mathrm{~N}$ pour le dosage des acides aminés ou solubilisé dans du Solvène 350 (Packard) pour la mesure de la radioactivité. Le dosage de la leucine est effectué sur colonne échangeuse d'ions à l'aide d'un analyseur automatique sur lequel est intercalé en sortie de colonne un spectromètre à cellule d'anthracène permeftant de mesurer la radioactivité des acides aminés marqués. Les autres mesures de radioactivité sont réalisées par scintillation liquide.

Le calcul de la fraction des protéines du muscle synthétisée par unité de temps (vitesse de synthèse fractionnaire $K_{\mathrm{s}}$ en temps ${ }^{-1}$ ) est effectué à partir de la relation entre la radioactivité spécifique (RAS) du précurseur sous forme libre $F$ et la RAS des protéines $P$ dans un modèle simple du type précurseur produit :

$\frac{d P}{d t}=k_{8}(F-P) \quad$ (Zilversmit, Enteman and Fishler, 1943 ; Poole, 1971). 
Cette relation peut se résoudre par :

$P(t)=k_{s} e^{-k_{s} t} \int_{0}^{t} e^{k_{s} u} F(u) d u \quad$ (Martin et al., 1977).

Nous avons ajusté $F$ (approché par la RAS de la leucine libre dans le compartiment plasmatique ou dans le compartiment intracellulaire du muscle) à une fonction multiexponentielle :

$F(t)=\sum_{i} X_{i} e^{-\lambda_{i} t}$

La résolution devient :

$P(t)=k_{s} \sum_{i} \frac{X_{i}}{k_{s}-\lambda_{i}}\left(e^{-\lambda_{i} t}-e^{-k_{s} t}\right)$

$\mathrm{k}_{\mathrm{s}}$ est alors déterminé en ajustant les valeurs expérimentales de $\mathrm{P}$ aux valeurs calculées par cette relation (Zak, Martin et Blough, 1979).

\section{Résultałs.}

Résultats zootechniques. - La croissance des truites élevées à $18^{\circ} \mathrm{C}$ est significativement plus élevée que celle des truites acclimatées à $10^{\circ} \mathrm{C}$ : respectivement 1,5 et 1,1 p. 100 du poids vif/jour (tabl. 1). Cela semble principalement lié à l'augmentation

\section{TABLEAU 1}

Poids, croissance ef consommation des truites acclimatées à 10 ef $18{ }^{\circ} \mathrm{C}$

\begin{tabular}{ccccc}
\hline Température & $\begin{array}{c}\text { Poids vif } \\
\text { à l'abattage }(\mathrm{g})\end{array}$ & $\begin{array}{c}\text { Croissance } \\
(\mathrm{g} / \mathrm{j})\end{array}$ & $\begin{array}{c}\text { Consommation } \\
(\mathrm{g} / \mathrm{j})\end{array}$ & $\begin{array}{c}\text { Indice de } \\
\text { consom- } \\
\text { mation }\left(^{(1)}\right.\end{array}$ \\
\hline $10^{\circ} \mathrm{C}$ & $\begin{array}{c}107 \\
(5)\end{array}$ & $\begin{array}{c}1,2 \\
(0,2)\end{array}$ & $\begin{array}{c}1,6 \\
(0,4)\end{array}$ & 1,3 \\
$18^{\circ} \mathrm{C}$ & $\begin{array}{c}(1,8 \\
2,0\end{array}$ & $(0,8)$ & $(0,7)$ & 1,1 \\
\hline $\begin{array}{c}\text { Signification } \\
\text { de la comparaison } \\
10 / 180^{\circ} \mathrm{C}\left({ }^{(2)}\right.\end{array}$ & $* * *$ & $*$ & $* * *$ & $*$ \\
\hline
\end{tabular}

(1) Consommation ( $g$ de poids frais/j)/croissance ( $g$ poids vif/j).

(2) Test t-student : * significatif au seuil 1 p. 100.

( ) Ecart-fype.

*** significatif au seuil 1 p. 1000 .

de la taille du muscle (la fraction prélevée représente 21 et 23 p. 100 du poids vif à 10 et $18{ }^{\circ} \mathrm{C}$ ), et à l'augmentation de la feneur en protéines du muscle (146 mg et $157 \mathrm{mg}$ de protéines $/ g$ muscle à 10 et $18^{\circ} \mathrm{C}$ ), la teneur en leucine de ces protéines étant inchangée $(\simeq 8,5 \mathrm{~g} / 16 \mathrm{gN})$. La consommation est plus forte à $18^{\circ} \mathrm{C}$ qu'à $10^{\circ} \mathrm{C}$, respective- 
TABLEAU 2

Teneurs en protéine ef leucine libre ou protéique du muscle ef du plasma des truites acclimatées à 10 ef $18{ }^{\circ} \mathrm{C}$

\begin{tabular}{|c|c|c|c|c|c|}
\hline Température & $\begin{array}{c}\text { Muscle } \\
\text { prélevé } \\
\text { (p. } 100 \text { poids vif) }\end{array}$ & $\begin{array}{c}\text { Teneur en } \\
\text { protéine du } \\
\text { muscle (mg/g) }\end{array}$ & $\begin{array}{c}\text { Teneur en } \\
\text { leucine des } \\
\text { protéines du } \\
\text { muscle }(\mathrm{g} / 16 \mathrm{gN})\end{array}$ & $\begin{array}{c}\text { Teneur en } \\
\text { leucine libre } \\
\text { plasmatique } \\
(\mu \text { mole } / g)\end{array}$ & $\begin{array}{l}\text { Teneur en } \\
\text { leucine libre } \\
\text { du muscle } \\
\text { (umole/g) }\end{array}$ \\
\hline $\begin{array}{l}10^{\circ} \mathrm{C} \\
18^{\circ} \mathrm{C}\end{array}$ & $\begin{array}{l}21 \\
(1,6) \\
24 \\
(1), 1\end{array}$ & $\begin{array}{l}146 \\
157\end{array}$ & $\begin{array}{l}8,4 \\
8,7\end{array}$ & $\begin{array}{c}1,3 \\
(0,4) \\
0,9 \\
(0,3)\end{array}$ & $\begin{array}{c}1,1 \\
(0,4) \\
0,7 \\
(0,3) \\
\end{array}$ \\
\hline $\begin{array}{l}\text { Signification des } \\
\text { comparaisons } \\
10 / 18^{\circ} \mathrm{C}(1)\end{array}$ & $*$ & * & $*$ & * & $*$ \\
\hline
\end{tabular}

(1) Test t-Student : * significatif au seuil 1 p. 100.

() Ecart-type.

ment 1,7 et 1,5 p. 100 du poids vif, d'où un indice de consommation plus faible chez les truites accli matées à $18^{\circ} \mathrm{C}$ qu'à $10^{\circ} \mathrm{C}$.

La concentration en leucine du plasma est significativement plus élevée que celle du muscle ef ces concentrations sont significativement plus faibles à $18^{\circ} \mathrm{C}$ qu'à $10^{\circ} \mathrm{C}$ (tabl. 2).

Evolution de la leucine ${ }^{14} \mathrm{C}(U)$ dans le plasma et le muscle après l'injection.

a) Sous forme libre. - La RAS de la leucine dans le plasma est plus élevée à $18^{\circ} \mathrm{C}$ qu'à $10^{\circ} \mathrm{C}$ dans les premiers temps après l'injection et plus faible à $18^{\circ} \mathrm{C}$ dans les temps longs qu'à $10^{\circ} \mathrm{C}$; elle diminue donc plus rapidement à $18^{\circ} \mathrm{C}$ qu'à $10^{\circ} \mathrm{C}$ (fig. 1).

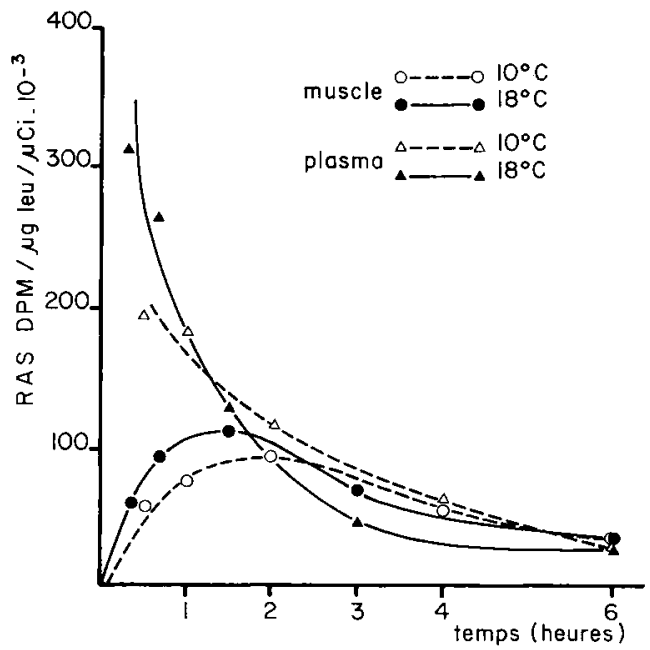

FIG. 1. - Evolution de la radioactivité spécifique (RAS) de la leucine libre du plasma ef du muscle de truites acclimatées $\dot{a} 10$ et $18^{\circ} \mathrm{C}$.

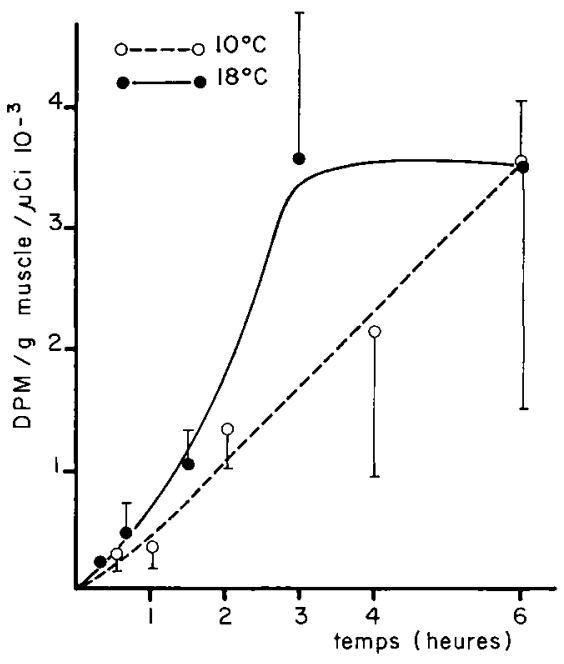

FIG. 2. - Evolution de la leucine ${ }^{14} \mathrm{C}(U)$ incorporée dans les protéines du muscle de truites acclimotées à 10 ef $18^{\circ} \mathrm{C}$. 
Les RAS de la leucine libre musculaire, plus élevées chez les truites acclimatées à $18^{\circ} \mathrm{C}$ qu'à $10^{\circ} \mathrm{C}$ dans les temps courts après l'injection, augmentent jusqu'à un maximum situé $2 \mathrm{~h}$ après l'injection à $10^{\circ} \mathrm{C}$ et $1 \mathrm{~h} 30$ à $18^{\circ} \mathrm{C}$, puis diminuent lentement et sont voisines dans les temps longs après l'injection (Fauconneau, 1980).

b) Sous forme liée aux protéines du muscle. - Les radioactivités des protéines musculaires des truites acclimatées à $18^{\circ} \mathrm{C}$ et $10^{\circ} \mathrm{C}$, non significativement différentes, augmentent et atteignent des maxima respectivement au voisinage des 3 et $6 \mathrm{~h}$ après l'injection (fig. 2). Ces augmentations de radioactivité s'ajustent bien à une fonction puissance, car les valeurs dans les premiers temps après l'injection sont faibles.

Au niveau du muscle entier la radioactivité totale liée aux protéines représente $6 \mathrm{~h}$ après l'injection, 8,6 p. 100 de la dose injectée à $10^{\circ} \mathrm{C}$ et 10,8 p. 100 à $18^{\circ} \mathrm{C}$.

Calcul des vifesses de synthèse des protéines du muscle. - La fraction des protéines du muscle synthétisée par jour $\mathrm{k}_{\mathrm{s}}$ est plus importante chez les truites acclimatées à $18^{\circ} \mathrm{C}$ que chez celles adaptées à $10^{\circ} \mathrm{C}(+34$ p. 100), si l'on assimile le pool précurseur au pool libre extracellulaire (représenté par le plasma) (tabl. 3). Lorsque le

TABLEAU 3

Fraction des protéines synthétisées par jour $k_{s}$ (p. 100/j) dans le muscle de truifes acclimatées d 10 ef 18 o C

\begin{tabular}{|c|c|c|c|}
\hline \multirow{2}{*}{ Température } & \multicolumn{3}{|c|}{ Compartiment utilisé pour le calcul de $k_{\delta}$} \\
\hline & Plasma & Libre & Intracellulaire \\
\hline $\begin{array}{l}10^{\circ} \mathrm{C} \\
18^{\circ} \mathrm{C}\end{array}$ & $\begin{array}{c}0,9 \\
(1,1) \\
1,2\end{array}$ & $\begin{array}{c}1,7 \\
(2,2) \\
1,7\end{array}$ & $\begin{array}{c}1,9 \\
(1,7) \\
1,8\end{array}$ \\
\hline $18^{\circ} \mathrm{C}$ & $\begin{array}{c}1,2 \\
(1,6)\end{array}$ & $\begin{array}{c}1,7 \\
(1,4)\end{array}$ & $\begin{array}{c}1,8 \\
(1,6)\end{array}$ \\
\hline
\end{tabular}

() Ecart-type.

calcul est effectué avec le pool libre tissulaire ou ce même pool corrigé en tenant compte de l'espace extracellulaire du muscle de poisson (Houston et al., 1968 ; Cooperstein et Lazarow, 1969), afin d'obtenir un pool intracellulaire, les estimations ne sont pas significativement différentes à 10 et à $18^{\circ} \mathrm{C}$, mais sont 1,5 à 2 fois plus élevées que celles obtenues à partir du plasma. La signification des ajustements réalisés est la meilleure avec ce dernier type d'estimation.

Quantités totales de protéines synthétisées par la masse musculaire. - Ces quantités estimées à partir des valeurs de vitesse de synthèse des protéines obtenues avec le pool intracellulaire sont de $166 \mathrm{mg} /$ jour $( \pm 130)$ à $10^{\circ} \mathrm{C}$ et de $198 \mathrm{mg} / \mathrm{jour}( \pm 178)$ à $18^{\circ} \mathrm{C}$. Les quantités de proféines fixées par le muscle (croissance) sont calculées en considérant que 60 p. 100 de la croissance du poisson est due au muscle (Luquet, 1971) et que les teneurs en protéines de ce gain de poids du muscle sont identiques à celles du muscle : $87 \mathrm{mg} /$ jour à $10^{\circ} \mathrm{C}$ ef $141 \mathrm{mg} / \mathrm{jour}$ à $18^{\circ} \mathrm{C}$. Les quantités de protéines dégradées représentent donc 48 p. 100 et 28 p. 100 d 10 et $18^{\circ} \mathrm{C}$ des quantités de protéines réellement synthétisées. 


\section{Discussion.}

Les truites élevées dans les conditions de notre expérience présentent une croissance musculaire plus importante à $18^{\circ} \mathrm{C}$ qu'à $10^{\circ} \mathrm{C}$. En outre, la taille du compartiment protéique du muscle est plus grande pour un même poids de muscle à $18{ }^{\circ} \mathrm{C}$ qu'à $10^{\circ} \mathrm{C}$. L'écart constaté dans les teneurs en leucine libre du plasma et du muscle entre 10 et $18^{\circ} \mathrm{C}$ confirme celui observé dans la fraction a aminée libre du plasma entre 8 et $18{ }^{\circ} \mathrm{C}$ (Fauconneau et Luquet, 1979).

Utilisation métabolique de la leucine par le muscle. - La leucine est métabolisée plus vite au niveau du poisson entier à $18^{\circ} \mathrm{C}$ qu'à $10^{\circ} \mathrm{C}$. L'entrée de leucine dans le muscle semble se faire plus rapidement à $18^{\circ} \mathrm{C}$ qu'à $10^{\circ} \mathrm{C}$ (référence au temps d'apparition des maxima de la RAS de la leucine libre musculaire), les valeurs plus élevées de RAS à $18^{\circ} \mathrm{C}$ qu'à $10^{\circ} \mathrm{C}$ dans les temps courts étant principalement la conséquence des teneurs en leucine libre du muscle plus faibles à $18^{\circ} \mathrm{C}$ qu'à $10^{\circ} \mathrm{C}$. La diminution de la RAS dans le muscle, similaire à celle du plasma dans les temps longs, met en évidence l'équilibre entre ces deux compartiments. Tout se passe comme si dans les temps longs après l'injection l'évolution du marqueur dans le plasma était le reflet de celle du muscle, compte tenu de la masse que représente celui-ci dans l'organisme (55 à 65 p. 100 du poids vif chez la truite). L'analyse de l'évolution de la leucine ${ }^{14} \mathrm{C}(\mathrm{U})$ libre tissulaire du muscle comparée à celle du plasma suggère une entrée lente de leucine dans le muscle, une accumulation de cet acide aminé dans le muscle et un relargage de cet acide aminé par le muscle dans les temps longs après l'injection.

L'utilisation de la leucine pour la synthèse protéique semble faible car les quantités de radioactivité retrouvées dans la musculatu re totale sont peu importantes comparées à la masse que représente ce tissu. Cette utilisation semble plus importante à $18^{\circ} \mathrm{C}$ qu'à $10^{\circ} \mathrm{C}$ mais elle est non - significativement différente entre ces deux températures.

Synthèse protéique dans le muscle de truite. - Les estimations de vitesse de synthèse des protéines du muscle, bien qu'imprécises, confirment la faible utilisation des acides aminés par cette voie. La fraction des protéines du muscle synthétisée par jour est faible (moins de 2 p. 100/jour). Cela est en accord avec les résultats obtenus chez le muge (Haschemeyer et Smith, 1979), chez le baliste (Haschemeyer, Persell ef Smith, 1979) et chez différentes espèces de poissons du Pacifique (Smith ef al., 1980) par la méthode d'infusion. Ces auteurs extrapolent les valeurs obtenues à 28 ou $30^{\circ} \mathrm{C}$ à la température de $37^{\circ} \mathrm{C}$ (température du rat) en tenant comple d'un $Q_{10}$ de 2,5 pour ce type de réaction. Un raisonnement analogue appliqué à nos mesures conduit à des valeurs extrapolées ( 3 à 5 p. 100/jour) proches de celles obtenues dans le muscle de Rat (Millward et Waterlow, 1978), de Souris (Earl et al., 1978) et de poulet (Laurent ef al., 1978) adultes. Mais pour des jeunes rats en croissance, les valeurs de fraction de protéines synthétisées par jour sont 2 à 3 fois plus élevées (10 à 15 p. 100/jour) que nos valeurs extrapolées.

Selon la nomenclature utilisée par Millward et al., (1976) la capacité de synthèse des protéines (estimée par le rapport RNA/protéines), du muscle de truite est semblable à celle du muscle de rat : respectivement $6,8 \mathrm{mg} \mathrm{RNA} / \mathrm{g}$ protéines (Luquet, 1971) et 
$11,6 \mathrm{mg} \mathrm{RNA} / \mathrm{g}$ protéines (Millward et al., 1976), mais l'efficacité brute de la synthèse protéique (quantité de protéines synthétisées/g RNA/jour) est nettement plus faible chez la truite (2 à $3 \mathrm{~g}$ protéines $/ \mathrm{g}$ RNA/jour) que chez le rat $(13,7 \mathrm{~g}$ protéines $/ \mathrm{g}$ RNA/jour). Cette particularité peut être expliquée par la régulation du métabolisme protéique du muscle. Chez les mammifères le muscle est le compartiment le plus sensible du point de vue renouvellement des protéines aux divers stimuli externes ou pathologiques. C'est le diabète qui affecte le plus le muscle à ce niveau (Pain et Garlick, 1974) y compris l'efficacité de la synthèse des protéines (Millward et Waterlow, 1978). Or, du point de vue du métabolisme glucidique, la truite a un comportement de type « diabétique » (Cowey ef al., 1977) en partie en raison d'une faible activité biologique de l'insuline de Poisson (Freychet, 1976). Ce type de déviation dans la régulation hormonale peut intervenir sur le renouvellement des protéines du muscle sans que l'on puisse discerner si cette déviation est une conséquence directe de la poecilothermie (l'équilibre insuline/glucagon étant très sensible à la température d'acclimatation, Umminger, 1978).

Renouvellement des protéines du muscle. - La croissance du muscle peut avoir une forte influence sur l'estimation de la fraction des protéines synthétisées (Zak, Martin et Blouch, 1979), l'utilisation de cette correction conduit à des renouvellements des protéines du muscle de 0,8 à 0,5 p. $100 /$ jour respectivement à 10 et $18{ }^{\circ} \mathrm{C}$. Les temps de renouvellement correspondants seraient de 110 jour à $10^{\circ} \mathrm{C}$ et 200 jours à $18^{\circ} \mathrm{C}$ (au minimum 40 et 45 jours en tenant compte de la variabilité des estimations), donc plus élevées à $18^{\circ} \mathrm{C}$ qu'à $10^{\circ} \mathrm{C}$ comme le suggéraient Somero ef Doyle (1973). Ces valeurs sont très faibles, mais surtout la participation du processus de synthèse des protéines à leur renouvellement est faible comparée à la part des protéines synthétisées qui sont fixées. Des résultats similaires auraient été obtenus également chez la truite (Smith, en préparation, cité par Haschemeyer, Persell et Smith, 1979).

Cette originalité du poisson est à rapprocher du déterminisme de la croissance chez ces animaux. Par l'étude des teneurs en acides nucléiques, il a été montré que l'hyperplasie contribue toujours pour plus de 70 p. 100 à la croissance pondérale du muscle, $y$ compris chez les poissons âgés (plus de 90 p. 100) (Luquet et Durand, 1970). Ce phénomène, valable pour les autres tissus du poisson à un moindre degré (Luquet, 1971 ; Luquet et Hannequart, 1974) permet de penser que la croissance du poisson est infinie. De plus, la croissance du muscle au niveau macroscopique est due principalement à l'augmentation du nombre de fibres (Weatherley, Gill ef Rogers, 1979) contrairement à ce que l'on peut observer chez les mammifères (Dauzier, 1976). Ces particularités dans la croissance du muscle et dans son déterminisme expliquent peut-être la relation différente observée chez la truite par rapport aux mammifères entre le renouvellement ef la fixation des protéines. Les valeurs élevées de vitesse de dégradation des protéines obtenues par Bouche (1975) par la méthode de décroissance chez des carpes en pleine croissance musculaire, pourraient également être reliées au processus de croissance.

\section{Conclusion.}

La leucine est métabolisée plus rapidement chez la truite acclimatée à $18^{\circ} \mathrm{C}$ qu'à 
$10^{\circ} \mathrm{C}$. La vitesse de synthèse des protéines musculaires chez la jeune truite est considérablement plus faible que chez le jeune mammifère (rat, agneau) et la température d'élevage du poisson n'a pas d'influence sur l'intensité de la synthèse protéique musculaire.

6e Réunion du groupe Développement I.N.R.A., Clermont-Ferrand/Theix, 22-23 mai 1980.

Remerciements. - Les dosages radiochromatographiques des acides aminés ont été faits par J. Prugnaud que nous remercions vivement.

\section{Références}

BOUCHE G., 1975. Recherches sur les acides nucléiques ef la proteosynthèse lors du jeâne prolongé chez la carpe. Th. Doct. Sci., Toulouse.

CASTILLA C., 1974. Influences de l'insuline, du glucagon ef du cortisol sur quelques aspects du métabolisme glucidique et protéique de la carpe. Th. Doct. $3^{\text {e }}$ Cycle, Toulouse.

COOPERSTEIN S. J., LAZAROW A., 1969. Fluid compartments of islet and other tissues of the toadfish Opsanus tou. Am. J. Physiol., 217, 1776-1783.

COWEY C. B., KNOX D., WALTON M. J., ADRON J. W., 1977. The regulation of neoglucogenesis by diet and insulin in rainbow trout (Salmo gairdneri). Br. J. Nutr., 38, 463-470.

DAS A. B., PROSSER C. L., 1967. Biochemical changes in tissues of goldfish acclimated to high and low temperatures. I Protein synthesis. Comp. Biochem. Physiol., 21, 449-467.

DAUZIER L., 1976. Diversity of muscular and adipose tissues : structure, growth and development, 3-36. In VERMOREL M., Energy metabolism of form animals. Proc. 7th Symp. EAAP, G. de Bussac, Clermont-Ferrand.

EARL C. A., LAURENT G. J., EVERETT A. W., BONNIN C. M., SPARROW M. P., 1978. Turnover rates of muscle protein in cardiac and skeletal muscles of dog, fowl, rat and mouse : turnover rate related to muscle function. Aust. J. exp. Biol. med. Sci., 56, 265-277.

FAUCONNEAU B., 1980. Synthèse protéique in vivo chez la truite arc-en-ciel (Salmo gairdneri R.). Métabolisme de la leucine. Influence de la température. Th. Doct. Ingén. I.N.A., Paris Grignon.

FAUCONNEAU B., LUQUET P., 1979. Effect of temperature rise on blood aminoacid and ammonia in rainbow trout (Salmo gairdneri R.) after feeding. Ann. Biol. anim. Bioch. Biophys., 19, 1063-1079.

FERRARA M., 1976. Synthèse protéique in vivo dans le muscle d'agneau au cours du développement. Détermination de la synthèse protéique du foie à l'aide du ${ }^{14} \mathrm{C}$ Lysyl tRNA lys. Th. Doct. $3^{\mathrm{e}}$ cycle, Strasbourg.

FREYCHET P., 1976. Interactions of polypeptide hormones with cell membrane specific receptors : studies with insulin and glucagon. Diabetologia, 12, 83-100.

HASCHEMEYER A. E. V., PERSELL R., SMITH M. A. K., 1979. Effect of temperature on protein synthesis in fish of the Galupagos and Perlas Islands. Comp. Biochem. Physiol., 64, 91-95.

HASCHEMEYER A. E. V., SMITH M. A. K., 1979. Protein synthesis in liver, muscle and gill of mullet (Mugil cephalus L.) in vivo. Biol. Bull., 156, 93-102.

HOUSTON A. H., REAVES R. S., MADDEN J. A., DE WILDE M. A., 1968. Environmental temperature and the body fluid systems of the fresh water teleost : I lonic regulation in thermally acclimated rainbow trout, Salmo gairdneri. Comp. Biochem. Physiol., 25, 563-581.

JACKIM E., LAROCHE G., 1973. Protein synthesis in Fundulus heteroclitus muscle. Comp. Biochem. Physiol., 44, 851-866.

LAURENT G. J., SPARROW M. P., BATES P. C., MILLWARD D. J., 1978. Turnover of muscle proteins in the fowl (Gallus domesticus). Biochem. J., 176, 393-405.

LUQUET P., 1971. Etude du développement chez la truite. Evolution de la teneur en acides nucléiques dans diverses fractions corporelles. Ann. Biol. anim. Bioch. Biophys., 11, 657-668.

LUQUET P., DURAND G., 1970. Evolution de la teneur en acides nucléiques de la musculature au cours de la croissance chez la truite Arc-en-ciel (Salmo gairdneri). Rôles respectifs de la multiplication ef du grandissement cellulaire. Ann. Biol. anim. Bioch. Biophys., 10, 481-492. 
LUQUET R., HANNEQUART M. H., 1974. Relations entre la longueur du poisson, le poids du cerveau et sa teneur en acides nucléiques chez la Carpe Royale. C. R. Acad. Sci. Paris, sér. D, 278, 3371-3374.

MARTIN A. F., RABINOWITZ M., BLOUGH R., PRIOR G., ZAK R., 1977. Measurement of half live of rat cardiac myosin heavy chain with leucyl tRNA used as precurser pool. J. biol. Chem., 252, 3422-3429.

MILLWARD D. J., GARLICK P. J., JAMES W. P. T., SENDER P. M., WATERLOW J. C., 1976. Protein turnover, 446-491, In COLE et al., Protein metabolism and nutrition, Butterworths, London.

MILLWARD D. J., WATERLOW J. C., 1978. Effect of nutrition on protein turnover in skeletal muscle. Fed. Proc., 37, 2283-2290.

NARAYANSINGH T., EALES J. G., 1975. Effect of thyroide hormones on L $1{ }^{14} \mathrm{C}$ Leucine incorporation into plasma and tissue protein of brook trout (Salvelinus fontinalis) and rainbow trout (Salmo gairdneri). Comp. Biochem. Physiol., 52, 399-405.

PAIN V. M., GARLICK P. J., 1974. Effects of streptozotocin diabetes and insulin treatment on the rate of protein synthesis in tissues of the rat in vivo. J. biol. Chem., 249, 4510-4514.

POOLE B., 1971. The kinetics of disappearance of labelled leucine from the free leucine pool of rat liver and its effect on the apparent furnover of catalase and other hepatic proteins. J. biol. Chem., 246, 6587-6591.

SMITH M. A. K., HASCHEMEYER A. E. V., 1974. Studies on protein metabolism and growth in fish. J. Fish. Res. Bd. Can., 21, 711-717.

SMITH M. A. K., MATHEWS R. W., HUDSON A. P., HASCHEMEYER A. E. V., 1980. Protein metabolism of tropical reef and pelagic fish. Comp. Biochem. Physiol., 65B, 415-418.

SOMERO G. N., DOYLE D., 1973. Température and rates of protein degradation in the fish Gillichthys mirabilis. Comp. Biochem. Physiol., 46, 463-474.

UMMINGER B. L., 1978. The role of hormones in the acclimation of fish to low temperatures. Naturwissentsch., 65, 144-150.

WATERLOW J. C., GARLICK P. J., MILLWARD D. J., 1978. Protein turnover in mammalian tissues and in the whole body. Elsevier North Holland, Biomed. Press, Amsterdam.

WEATHERLEY A. H., GILL. H. S., ROGERS S. C., 1979. Growth dynamics of muscle fibres, dry weight and condition in relation to somatic growth rate in yearling rainbow trout (Salmo gairdneri). Can. J. Zool., 57, 2385-2392.

ZAK R., MARTIN A. F., BLOUGH R., 1979. Assessment of protein turnover by use of radioisotopic tracers. Physiol. Rev., 59, 407-447.

ZILVERSMIT D. B., ENTEMAN C., FISHLER M. C., 1943. On the calculation of turnover time and turnover rate from experiments involving the use of labelling agents. J. gen. Physiol., 26, 235331. 